\title{
Strong nonlocality variations in a spherical mean-field dynamo
}

\author{
A. Brandenburg ${ }^{1,2,3,4}$ \& P. Chatterjee ${ }^{5}$ \\ 1 Laboratory for Atmospheric and Space Physics, University of Colorado, Boulder, CO 80303, USA \\ 2 JILA \& Dept. of Astrophysical and Planetary Sciences, University of Colorado, Boulder, CO 80303, USA \\ 3 Nordita, KTH Royal Institute of Technology and Stockholm University, 10691 Stockholm, Sweden \\ 4 Department of Astronomy, Stockholm University, 10691 Stockholm, Sweden \\ ${ }^{5}$ Indian Institute of Astrophysics, II Block Koramangala, Bengaluru-560034, India
}

August 11, 2018, Revision: 1.56

Key words stars: activity - Sun: activity - magnetic fields - magnetohydrodynamics (MHD)

To explain the large-scale magnetic field of the Sun and other bodies, mean-field dynamo theory is commonly applied where one solves the averaged equations for the mean magnetic field. However, the standard approach breaks down when the scale of the turbulent eddies becomes comparable to the scale of the variations of the mean magnetic field. Models showing sharp magnetic field structures have therefore been regarded as unreliable. Our aim is to look for new effects that occur when we relax the restrictions of the standard approach, which becomes particularly important at the bottom of the convection zone where the size of the turbulent eddies is comparable to the depth of the convection zone itself. We approximate the underlying integrodifferential equation by a partial differential equation corresponding to a reaction-diffusion type equation for the mean electromotive force, making an approach that is nonlocal in space and time feasible under conditions where spherical geometry and nonlinearity are included. In agreement with earlier findings, spatio-temporal nonlocality lowers the excitation conditions of the dynamo. Sharp structures are now found to be absent. However, in the surface layers the field remains similar to before.

\section{Introduction}

The solar convection zone $(\mathrm{CZ})$ is strongly stratified not just in the sense that the density contrast is enormous, but also in the sense that the pressure scale height $H_{\mathrm{p}}$ varies strongly: from $150 \mathrm{~km}$ at the top of the $\mathrm{CZ}$ to about $50 \mathrm{Mm}$ at the bottom (Stix 2002). Given that the size of the turbulent eddies is expected to be proportional to $H_{\mathrm{p}}$, this change in $H_{\mathrm{p}}$ corresponds to a 300 fold increase in the typical scale of the turbulent eddies. It is unclear whether this change in the turbulence properties can just be translated to a corresponding position-dependent change of the related turbulent transport coefficients (e.g., turbulent diffusivity and $\alpha$ effect), as is usually assumed (e.g., Pipin 2008, 2017; Pipin \& Kosovichev 2011), or whether one should expect some more subtle effects as a result of strong stratification. In the former case, turbulent transport can be described by just a local Fickian diffusion law where the turbulent flux of a passive scalar, for example, is proportional to the gradient of the mean concentration and a diffusion coefficient that is given by the product of the local values of turbulent velocity and correlation length (Parker 1979; Rüdiger \& Hollerbach 2004). The latter is usually assumed to be a multiple of the local pressure scale height, and the turbulent velocity can be estimated from stellar mixing length theory (Krivodubskii 1984). The alternative to the local Fickian diffusion law is a nonlocal formulation, in which the turbulent flux of passive scalar concentration is given by a convolution with an integral kernel (Rädler 1976). Such a formulation might have unexplored effects when applied to solar dynamo theory, where the turbulent concentration flux in the Fickian diffusion law corresponds to the mean electromotive force in the mean-field induction equation. It is therefore necessary to investigate the effects of a strong scale height change in a system resembling the solar dynamo, but that is simple enough so that one has a chance to isolate the consequences of individual effects.

In the standard mean-field prescription in terms of $\alpha$ effect and turbulent diffusivity $\eta_{\mathrm{t}}$, the mean electromotive force $\overline{\mathcal{E}}$ is given by (Krause \& Rädler 1980)

$$
\overline{\mathcal{E}}=\alpha \overline{\boldsymbol{B}}-\eta_{\mathrm{t}} \mu_{0} \overline{\boldsymbol{J}},
$$

where anisotropies have been ignored. Here $\overline{\boldsymbol{B}}$ is the mean magnetic field, $\overline{\boldsymbol{J}}=\boldsymbol{\nabla} \times \boldsymbol{B} / \mu_{0}$ is the mean current density, and $\mu_{0}$ is the vacuum permeability. However, Equation (11) becomes invalid when the mean field shows variations on short time and length scales (Brandenburg et al. 2008a, Hubbard \& Brandenburg 2009). In that case the multiplications with coefficients $\alpha$ and $\eta_{\mathrm{t}}$ must be replaced by a convolutions with integral kernels $\hat{\alpha}$ and $\hat{\eta}_{\mathrm{t}}$. In practice such an approach is cumbersome, because one has to store the values of the mean fields at all past times at all positions. An easier 
way to deal with this is to turn the underlying integrodifferential equation into a partial differential equation. This can be done in sufficiently simple cases. An example that has previously been explored in this context is the telegraph equation approach, where one replaces $\overline{\mathcal{E}}$ by $\left(1+\tau \partial_{t}\right) \overline{\mathcal{E}}$ (Blackman \& Field 2002, 2003). Here $t$ is time and $\tau$ is some relevant relaxation time that is of the order of the turnover time of the turbulence; see Brandenburg et al. (2004). In spectral space, this corresponds to an integral kernel of the form $(1-i \omega \tau)^{-1}$, where $\omega$ is the frequency; see Hubbard \& Brandenburg (2009). The spatial part of the integral kernel is known to be a Lorentzian in spectral space (Brandenburg et al. 2008a), i.e., $\left(1+k^{2} / k_{\mathcal{E}}^{2}\right)^{-1}$, where $k_{\mathcal{E}}$ is a relevant wavenumber that is expected to be proportional to the wavenumber $k_{\mathrm{f}}$ of the energy-carrying eddies with a scale factor $a_{\mathcal{E}}=k_{\mathrm{f}} / k_{\mathcal{E}}$. This means that $\overline{\mathcal{E}}$ should be replaced by $\left(1-\ell^{2} \nabla^{2}\right) \overline{\mathcal{E}}$. Here $k$ is the wavenumber. Recent work by Rheinhardt \& Brandenburg (2012) has shown that the combination of both spatial and temporal effects at the same time yields

$$
\left(1+\tau \frac{\partial}{\partial t}-\ell^{2} \nabla^{2}\right) \overline{\mathcal{E}}=\alpha \overline{\boldsymbol{B}}-\eta_{\mathrm{t}} \mu_{0} \overline{\boldsymbol{J}}
$$

where $\ell \equiv k_{\mathcal{E}}^{-1}$ characterizes the length scale on which nonlocality becomes important. This equation has to be solved simultaneously with the usual mean-field dynamo equation. Obviously, this nonlocal formulation in space and time becomes equal to the local one in the limit $\tau \rightarrow 0$ and $\ell \rightarrow 0$. When applied to the Sun, we must expect the $\tau$ and $\ell$ terms to become important at the bottom of the $\mathrm{CZ}$ where the turnover time and the correlation length are large. When $\ell$ becomes large, the effect of the mean magnetic field on $\overline{\mathcal{E}}$ becomes reduced by what corresponds to a Lorentzian $1 /\left(1+\ell^{2} k^{2}\right)$ in Fourier space, i.e., not only the $\alpha$ effect but also turbulent diffusivity becomes progressively weaker in deeper layers of the Sun.

The aim of this paper is to assess the significance of a strong vertical variation of the degree of nonlocality in space and time on dynamo models that have stratification and rotation profiles similar to those expected for the Sun. We adopt a simple mixing length prescription through which rms velocity as well as correlation length and correlation time depend in power law fashion on the depth below the surface.

\section{Aspects of the model}

In this section, we motivate a number of aspects that should, on physical grounds, be included in a solar mean-field model. We begin by presenting first the basic equations, and then discuss several profile functions that characterize a model of the solar dynamo.

\subsection{Dynamo equations}

In this paper, we ignore temporal changes of the mean flow and consider only the changes of the mean magnetic field $\overline{\boldsymbol{B}}$, whose evolution is given by

$$
\partial \overline{\boldsymbol{A}} / \partial t=\overline{\boldsymbol{U}} \times \overline{\boldsymbol{B}}+\overline{\mathcal{E}}-\eta \mu_{0} \overline{\boldsymbol{J}}
$$

where $\overline{\boldsymbol{U}}$ is a prescribed mean flow, $\overline{\boldsymbol{B}}=\boldsymbol{\nabla} \times \overline{\boldsymbol{A}}$ is the mean magnetic field expressed in terms of the vector potential $\overline{\boldsymbol{A}}$, and $\mu_{0}$ is the vacuum permeability. The mean flow includes the differential rotation, i.e., $\bar{U}_{\phi}=$ $r \sin \theta \Omega(r, \theta)$, and in general also meridional circulation, which is here, however, neglected. Meridional circulation is particularly important in models with separated induction zones (Choudhuri et al. 1995; Dikpati \& Charbonneau 1999; Chatterjee et al. 2004). However, in models with overlapping induction zones, as in the present case, meridional circulation usually just increases the critical dynamo number, but does not change significantly the shape of the eigenfunction (Rädler 1986).

\subsection{Nonlocality in earlier work}

Previous studies using the test-field method applied to the steady state $(\omega=0)$ suggest that $\ell=a_{\eta} / k_{\mathrm{f}}$, where $a_{\eta}$ is a dimensionless parameter that is usually in the range 0.5-1 (Brandenburg et al. 2008a, 2009; Mitra et al. 2009), but it can be as low as 0.2 if there is shear (Madarassy \& Brandenburg 2010). Furthermore, in the unsteady case, the test-field method suggests that $\mathrm{St}=\tau u_{\mathrm{rms}} k_{\mathrm{f}}$ is in the range 1.4-2.0 (Hubbard \& Brandenburg 2009). Writing now Equation (2) as an evolution equation, we have

$$
\frac{\partial \overline{\mathcal{E}}}{\partial t}=\tau^{-1}\left(\alpha \overline{\boldsymbol{B}}-\eta_{\mathrm{t}} \mu_{0} \overline{\boldsymbol{J}}-\overline{\mathcal{E}}\right)+\eta_{\mathcal{E}} \nabla^{2} \overline{\mathcal{E}}
$$

where $\eta_{\mathcal{E}}=a_{\mathcal{E}}^{2} \eta_{\mathrm{t}}$ is a diffusion coefficient in the evolution equation for $\overline{\mathcal{E}}$, which is expected to scale like the turbulent magnetic diffusivity $\eta_{\mathrm{t}} \approx \frac{1}{3} \tau u_{\mathrm{rms}}^{2}$. Assuming furthermore that $\tau=\mathrm{St} / u_{\mathrm{rms}} k_{\mathrm{f}}$, where $\mathrm{St}$ is the Strouhal number (expected to be of the order of unity; see Sur et al. 2008), we have $a_{\mathcal{E}}=3 a_{\mathcal{E}}^{2} /$ St.

In this paper we examine the effects of nonlocality in a spherical shell dynamo. Recent work using simultaneous nonlocality in space and time has shown that St $=0.6-1.2$ and $a_{\mathcal{E}}=0.6-0.8$ for $\operatorname{Re}_{M} \approx 60$ (Rheinhardt \& Brandenburg 2012), corresponding to $a_{\mathcal{E}}=1.5-3$.

\subsection{Nonlinearity}

It should be noted that in the present approach the incorporation of nonlinear feedbacks does not require any special consideration. Both dynamical and algebraic quenching (see Brandenburg \& Subramanian 2005a for a review) can be applied in the usual sense. This is remarkable, because the original formalism in terms of 
integral kernels is a standard concept in linear response theory and thus not readily applicable to the nonlinear regime; see the discussion in Rheinhardt \& Brandenburg (2012).

At the simplest level we adopt just algebraic $\alpha$ quenching, by which the local value of $\alpha$ is suppressed locally proportional to a function

$$
f_{\alpha}(\overline{\boldsymbol{B}})=\frac{1}{1+Q_{\alpha} \overline{\boldsymbol{B}}^{2} / B_{\mathrm{eq}}^{2}},
$$

where $B_{\text {eq }}=\sqrt{\mu_{0} \rho} u_{\mathrm{rms}}$ is the equipartition field strength. In addition, we adopt the dynamical quenching model, in which we solve an evolution equation for a magnetic contribution to the $\alpha$ effect, i.e. $\alpha$ is the sum of kinetic and magnetic $\alpha$ effects, $\alpha=\alpha_{\mathrm{K}}+\alpha_{\mathrm{M}}$, where $\alpha_{\mathrm{M}}=(\tau / 3 \bar{\rho}) \overline{\boldsymbol{j} \cdot \boldsymbol{b}}$ is proportional to the current helicity, which, in the isotropic case, is proportional to the magnetic helicity of the small-scale field. The magnetic $\alpha$ effect, in turn, obeys an evolution equation that must be solved simultaneously. The equation for the evolution of $\alpha_{\mathrm{M}}$ is given by (e.g., Subramanian \& Brandenburg 2006)

$$
\frac{\partial \alpha_{\mathrm{M}}}{\partial t}=-2 \eta_{\mathrm{t}} k_{\mathrm{f}}^{2}\left(\frac{\overline{\mathcal{E}} \cdot \overline{\boldsymbol{B}}}{B_{\mathrm{eq}}^{2}}+\frac{\alpha_{\mathrm{M}}}{R_{\mathrm{m}}}\right)-\nabla \cdot \overline{\boldsymbol{F}}_{\alpha},
$$

where $\overline{\boldsymbol{F}}_{\alpha}=-\kappa_{\alpha} \nabla \alpha_{\mathrm{M}}$ is the turbulent-diffusive magnetic helicity flux, which was found to be the most important contribution (Hubbard \& Brandenburg 2011, 2012; Del Sordo et al. 2013). Here $\kappa_{\alpha}$ is a turbulent diffusion coefficient for the flux. In the following we assume $\kappa_{\alpha}=\eta_{\mathrm{t}}$ as a good approximation.

Chatterjee et al. (2011) have solved Equation (6) along with the mean field induction equation for an $\alpha \Omega$ interface dynamo in a spherical shell. They found that the time-latitude plot for the toroidal field shows signatures of what they called 'secondary dynamos' (see their Figs. 9 and 10). This phenomenon occurred both for vanishing magnetic helicity flux as well as for fluxes that were 'supercritical' in the sense that the fluxes alone would drive a dynamo of the type proposed by Vishniac \& Cho (2001); see Brandenburg \& Subramanian (2005b) for a mean-field model of such a dynamo. These magnetic helicity-driven dynamos were suspected to be excited due to the lack of nonlocality in their model. In this paper, we also run a similar nonlinear model along with the equation for $\partial \overline{\mathcal{E}} / \partial t$.

In practice, we combine both algebraic and dynamic $\alpha$ quenching and write

$$
\alpha=f_{\alpha}(\overline{\boldsymbol{B}})\left(\alpha_{\mathrm{K}}+\alpha_{\mathrm{M}}\right),
$$

Thus, the function $f_{\alpha}$ provides the ultimate limitation to $\alpha$ in the sense that $\alpha \rightarrow 0$ for strong magnetic fields.

\subsection{Model stratification}

Instead of using a realistic stratification from a solar model, we felt it more insightful to use a stratification based on simple approximations such as a polytropic one and to get in this way the radial dependence of density $\rho$. Furthermore, according to mixing length theory (Vitense 1953; Brandenburg 2016), the convective flux is, to a good approximation, equal to $\rho u_{\mathrm{rms}}^{3}$, where $u_{\mathrm{rms}}$ is the rms velocity of the turbulence. This relation fails near the bottom of the solar CZ, because there the radiative flux begins to dominate, so $u_{\text {rms }}$ would be overestimated. On the other hand, over sufficiently large ranges in radius $r$, it is not the flux $F$ that is constant, but the luminosity $4 \pi r^{2} F$, so $F$ should really be larger toward the bottom of the $\mathrm{CZ}$, so this would underestimate $u_{\mathrm{rms}}$. Here we ignore the two opposing effects and assume that they cancel each other to some extent.

We adopt an adiabatic stratification where the sum of local enthalpy, $h\left(=c_{p} T\right.$ for a perfect gas with specific heat at constant pressure $c_{p}$ ) and the gravitational potential $\Phi(=-G M / r$ for a point mass $M$, or $g z$ for plane-parallel layer with constant gravitational acceleration $g$ ) are constant. We assume that the mixing length $\ell$ is proportional to the pressure scale height, $H_{\mathrm{p}}=|\nabla \ln p|^{-1}$. We assume a polytropic index of $n=3 / 2$, so $\rho \sim \ell^{3 / 2}$, where $\ell$ is the mixing length, which is assumed to be proportional to the depth below the surface, i.e., $\ell=R-r \equiv k_{\mathrm{f}}^{-1}$ (cf. Canuto \& Mazzitelli 1991).

Using $\rho u^{3}=$ const, we have for the rms velocity the scaling

$$
u_{\mathrm{rms}} \sim k_{\mathrm{f}}^{1 / 2}
$$

which has its maximum at the surface. This implies that the correlation or turnover time has the scaling

$$
\tau \sim\left(u_{\mathrm{rms}} k_{\mathrm{f}}\right)^{-1} \sim k_{\mathrm{f}}^{-3 / 2},
$$

which has a maximum at the bottom of the $\mathrm{CZ}$, and similarly also the turbulent diffusivity $\eta_{\mathrm{t}}$ and the $\alpha$ effect,

$$
\eta_{\mathrm{t}} \sim \tau u_{\mathrm{rms}}^{2} \sim k_{\mathrm{f}}^{-1 / 2}, \quad \alpha \sim \Omega \ell \sim k_{\mathrm{f}}^{-1} .
$$

The local values of $\alpha$ and $\eta_{\mathrm{t}}$ would therefore reach a maximum at the bottom of the CZ (Krivodubskii 1984; Brandenburg \& Tuominen 1988). To avoid finite values of $\alpha$ and $\eta_{\mathrm{t}}$ at $r=r_{\mathrm{b}}$, we multiply $k_{\mathrm{f}}^{-1}$ by an additional profile function so that $\alpha$ goes smoothly to 0 at $r=r_{\mathrm{b}}$. Furthermore, both the memory term, $\tau \omega_{\text {cyc }} \sim k_{\mathrm{f}}^{-3 / 2}$, and the nonlocal term, $\left(k_{\mathrm{f}} R\right)^{-2} \sim k_{\mathrm{f}}^{-2}$, are maximum at the bottom. This makes the dynamo locally less efficient in the lower layers.

The $\alpha$ effect changes sign about the equator and is proportional to $\cos \theta$, while $\eta_{\mathrm{t}}$ is independent of $\theta$, so we write

$$
\alpha(r, \theta)=\frac{\alpha_{0} g(r) \cos \theta}{k_{\mathrm{f}}(r) R}, \quad \eta_{\mathrm{t}}(r)=\frac{\eta_{\mathrm{t} 0} g(r)}{\left(k_{\mathrm{f}}(r) R\right)^{1 / 2}} .
$$

Here $\alpha_{0}$ and $\eta_{\mathrm{t} 0}$ are coefficients that can be combined into a dynamo number,

$$
C_{\alpha}=\alpha_{0} R / \eta_{\mathrm{t} 0} \text {. }
$$




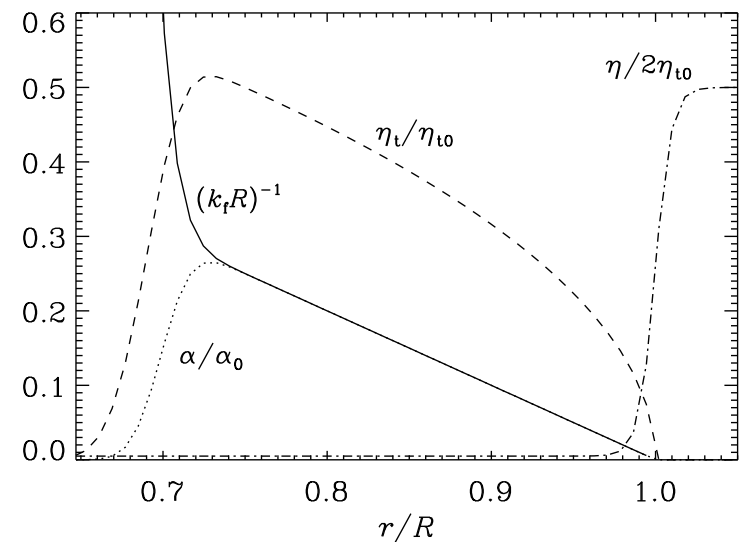

Fig. 1 Profiles of $\left(k_{\mathrm{f}} R\right)^{-1} \equiv 1-\ell / R, \alpha / \alpha_{0}$ and $\eta_{\mathrm{t}} / \eta_{\mathrm{t} 0}$.

In addition to a profile for $\eta_{\mathrm{t}}$, we also adopt a profile for the microscopic magnetic diffusivity $\eta$, which is assumed constant everywhere except in the outer parts where $\eta / \eta_{\mathrm{t} 0} \rightarrow 1$ to mimic a potential (current-free) magnetic field. Unlike the usual case of a local model, where only the combination $\eta+\eta_{\mathrm{t}}$ enters, the two are now different in that $\eta$ enters directly in the induction equation for the magnetic field while $\eta_{\mathrm{t}}$ enters in the evolution equation for the mean electromotive force.

In Figure 1 we plot radial profiles of $k_{\mathrm{f}}^{-1}$, as well as $\alpha / \alpha_{0}, \eta_{\mathrm{t}} / \eta_{\mathrm{t} 0}$, and $\eta / \eta_{\mathrm{t} 0}$

\subsection{Differential rotation profile}

We use a solar-like profile as a superposition of contributions from the tachocline (TC), a small positive radial differential rotation in the $\mathrm{CZ}$, and a sharp negative differential rotation in the near-surface shear layer (NSSL), i.e.,

$$
\Omega=\Omega_{0}\left[\Omega_{\mathrm{TC}}+c_{\Omega 2}\left(\Omega_{\mathrm{CZ}}+\Omega_{\mathrm{NSSL}}\right)\right],
$$

where

$$
\Omega_{\mathrm{TC}}=-\Theta^{+}\left(r-r_{\mathrm{b}}\right) C_{2}^{3 / 2}(\cos \theta)
$$

models the TC (the minus sign corresponds to equatorial acceleration),

$$
\Omega_{\mathrm{NSSL}}=-\Theta^{+}\left(r-r_{\mathrm{t}}\right)
$$

the NSSL, and

$$
\Omega_{\mathrm{CZ}}=\left(r-r_{\mathrm{b}}\right) \Theta^{+}\left(r-r_{\mathrm{b}}\right) \Theta^{-}\left(r-r_{\mathrm{t}}\right)\left(3-4 \cos ^{2} \theta\right)(16)
$$

the interior region in the $\mathrm{CZ}$. Here,

$$
C_{2}^{3 / 2}(\cos \theta)=-\frac{P_{3}^{1}(\cos \theta)}{\sin \theta}=\frac{3}{2}\left(5 \cos ^{2} \theta-1\right)
$$

is the Gegenbauer polynomial of degree $3 / 2$ and $P_{3}^{1}$ is the associated Legendre polynomial of degree 3 and order 1. In Figure 2 we compare contours of differential rotation with just the $\mathrm{TC}$ contribution $\left(c_{\Omega 2}=0\right)$ and with that using all contributions $\left(c_{\Omega 2}=1\right)$. In the following we quantify the strength of differential rotation by the nondimensional quantity $C_{\Omega}=\Omega_{0} R^{2} / \eta_{\mathrm{t} 0}$.
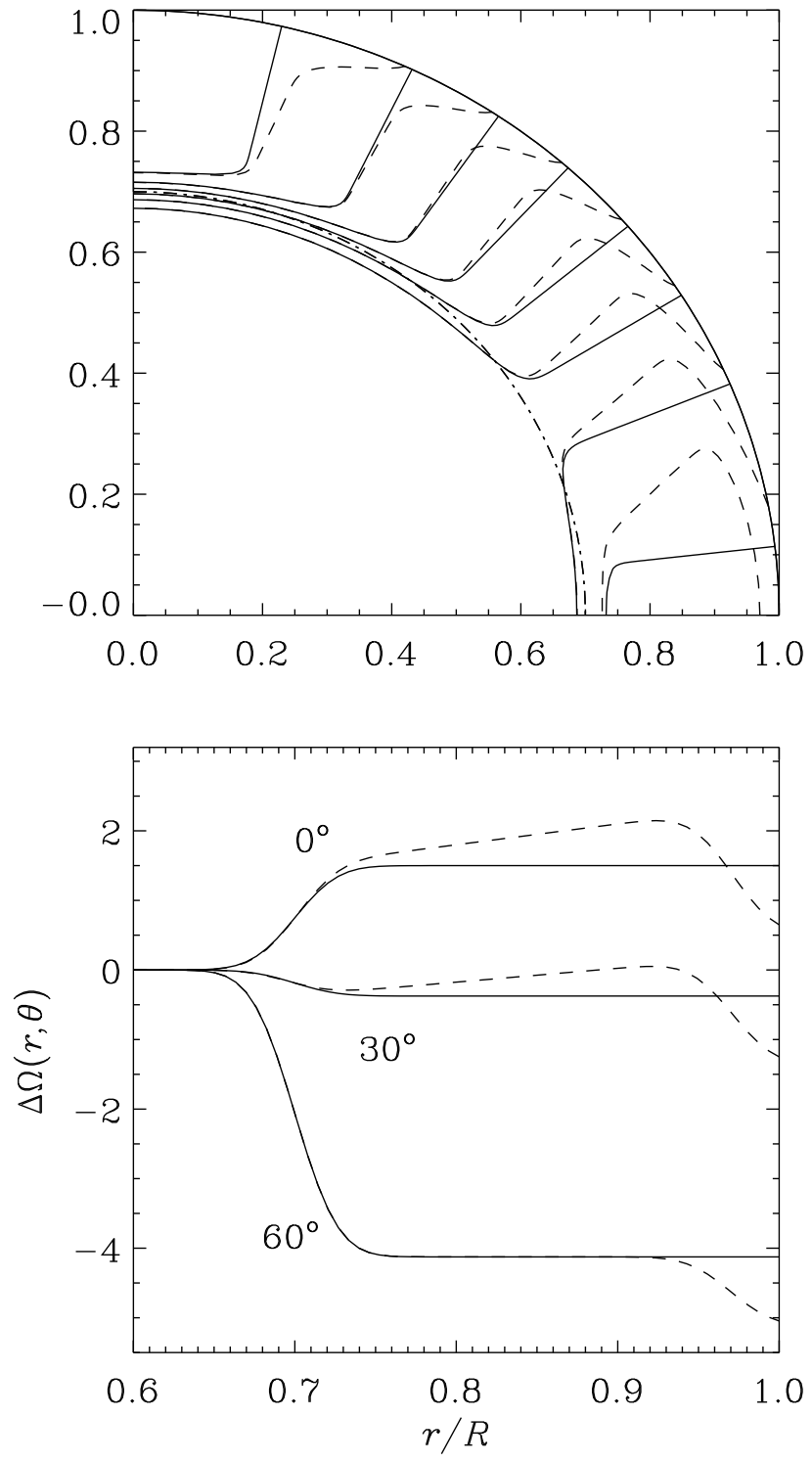

Fig. 2 Contours of differential rotation (upper panel) and radial profiles of angular velocity at three different latitudes (lower panel) from the simple model of Equation (13) with $c_{\Omega 2}=0$ (spoke-like contours; solid lines) and $c_{\Omega 2}=1$ (tilted contours with NSSL; dashed lines).

We use the PENCIL Cod 1 (revision 19,388 or later). We use spherical polar coordinates, $(r, \theta, \phi)$. We solve the equations in two dimensions $(\partial / \partial \phi=0)$ in $r_{\mathrm{i}} \leq$ $r \leq r_{\mathrm{o}}$, which is large enough to encompass the bottom of the CZ $\left(r=r_{\mathrm{b}}\right)$ and the outer radius of the sphere $(r=R)$. In the following, length is usually expressed in units of $R$.

\footnotetext{
1 http://github.com/pencil-code
} 
Table 1 Summary of runs mentioned in the text. Nonlinear runs are marked by nlin and figure numbers are given in the last column.

\begin{tabular}{lccrccccc} 
Run & St & $a_{\mathcal{E}}$ & \multicolumn{1}{c}{$D$} & $c_{\Omega 2}$ & nlin & $T_{\text {cyc }}$ & $B_{\text {rms }}$ & Fig(s) \\
\hline K1N & 1 & 1 & 500 & 1 & no & 0.526 & - & 3 \\
K1L & 0 & 0 & 4000 & 1 & no & 0.108 & - & 4 \\
K0N & 1 & 1 & 500 & 0 & no & 0.531 & - & {$[5$} \\
K0L & 0 & 0 & 4000 & 0 & no & 0.108 & - & 6 \\
Q1N & 1 & 1 & 100 & 1 & yes & 0.539 & 21.9 & $7[9]$ \\
Q1L & 0 & 0 & 2500 & 1 & yes & 0.289 & 0.44 & $8]$
\end{tabular}

\subsection{Model parameters}

The model geometry is defined by the following choice: $r_{\mathrm{i}}=0.55, r_{\mathrm{b}}=0.7, R=1$, and $r_{\mathrm{o}}=1.05$. We restrict ourselves to solving the equations in one quadrant of the meridional plane $(0 \leq \theta \leq \pi / 2$, and adopt a condition at the equator that selects only solutions that are antisymmetric about the equatorial plane.

Unless specified differently, we adopt for all radial profiles a width of $w=0.02$, which can still be reasonably well resolved with just 64 mesh points in the radial direction. In the latitudinal direction we take 96 mesh points.

In all calculations, we make the $\alpha \Omega$ approximation, i.e., we assume that the $\alpha$ tensor is finite only in its $\phi \phi$ component, so we can neglect the $\alpha$ effect in the generation of the toroidal field in comparison with the differential rotation. This means that the dynamo onset is only determined by one dynamo number, namely $D=C_{\alpha} C_{\Omega}$. This has the advantage that there is one parameter less to consider, although it is somewhat unrealistic.

\section{Results}

In the following, we vary the parameters St, $a_{\mathcal{E}}, D$, and $c_{\Omega 2}$. Our runs are summarized in Table 1 .

\subsection{Kinematic runs with NSSL}

In Figures 3 and 4 we compare nonlocal models (with $\partial \overline{\mathcal{E}} / \partial t$ equation included) and local ones (without $\partial \overline{\mathcal{E}} / \partial t$ equation) by showing field lines in the meridional plane together with a color-coded representation of the toroidal field. Note that with the $\partial \overline{\mathcal{E}} / \partial t$ equation, the cycle frequency is about 5 times lower than without this equation $\left(\mathrm{St}=a_{\mathcal{E}}=0\right)$, and also the excitation condition is about 5 times lower. This is in agreement with similar results in Cartesian geometry (Rheinhardt \& Brandenburg 2012).
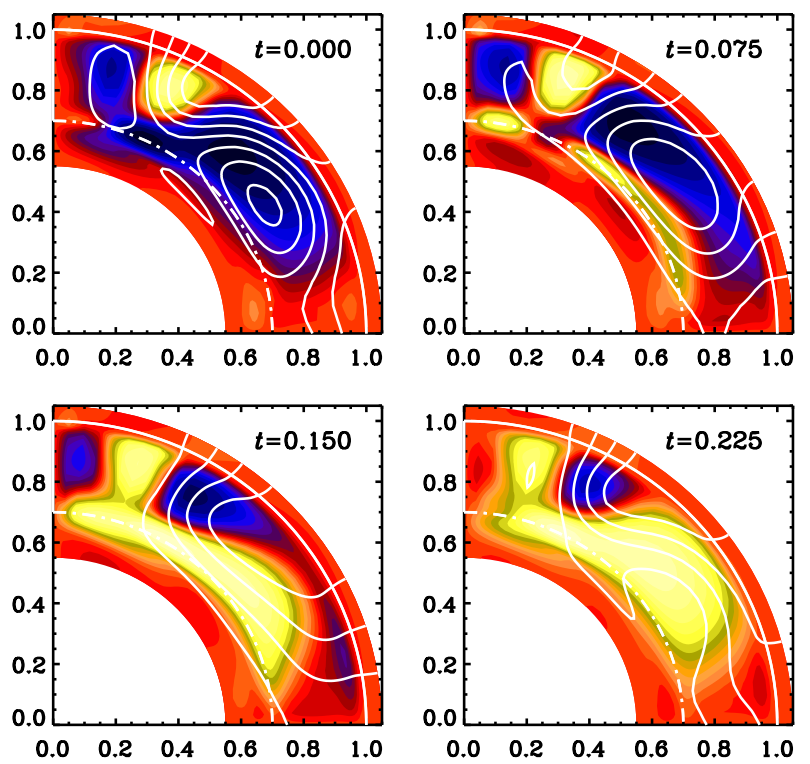

Fig. 3 Field lines in the meridional plane together with a color-coded representation of the toroidal field (dark/blue shades indicate negative values and light/yellow shades positive values). Evolution of the field structure for Run K1N with NSSL and $D=500$ (slightly supercritical, oscillatory), using the $\partial \overline{\mathcal{E}} / \partial t$ equation with $\mathrm{St}=1$ and $a_{\mathcal{E}}=1$.
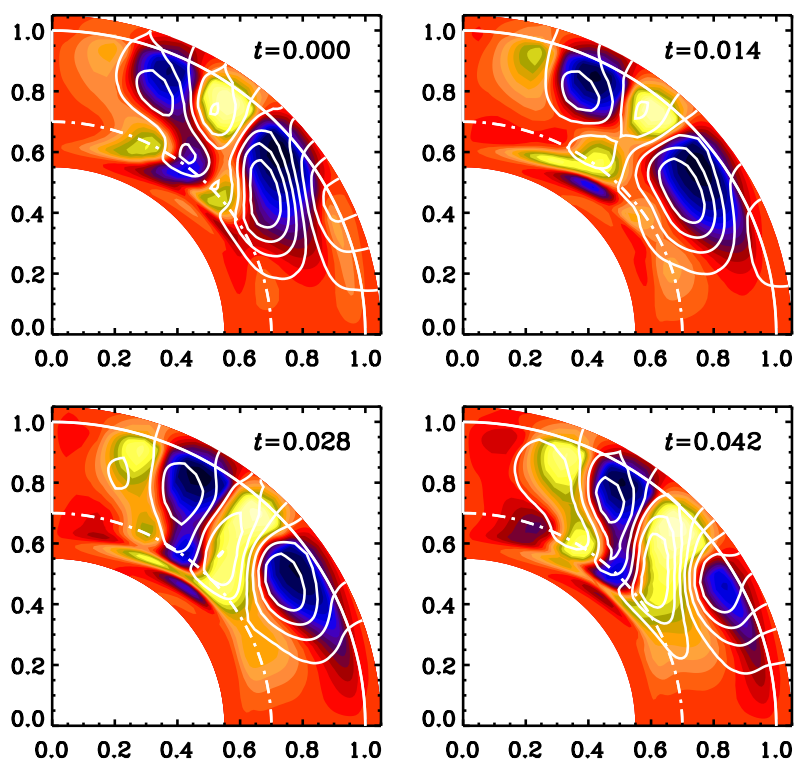

Fig. 4 Like Figure 3, but for Run K1L without the $\partial \overline{\mathcal{E}} / \partial t$ equation and $D=4000$ (slightly supercritical, oscillatory), using a local model, i.e., $\mathrm{St}=a_{\mathcal{E}}=0$.

\subsection{Kinematic runs without NSSL}

Let us now compare with the corresponding cases where the NSSL is omitted (i.e., $c_{\Omega 2}=0$ ). In Figures 5 and 6 we show again meridional cross-sections of the mag- 

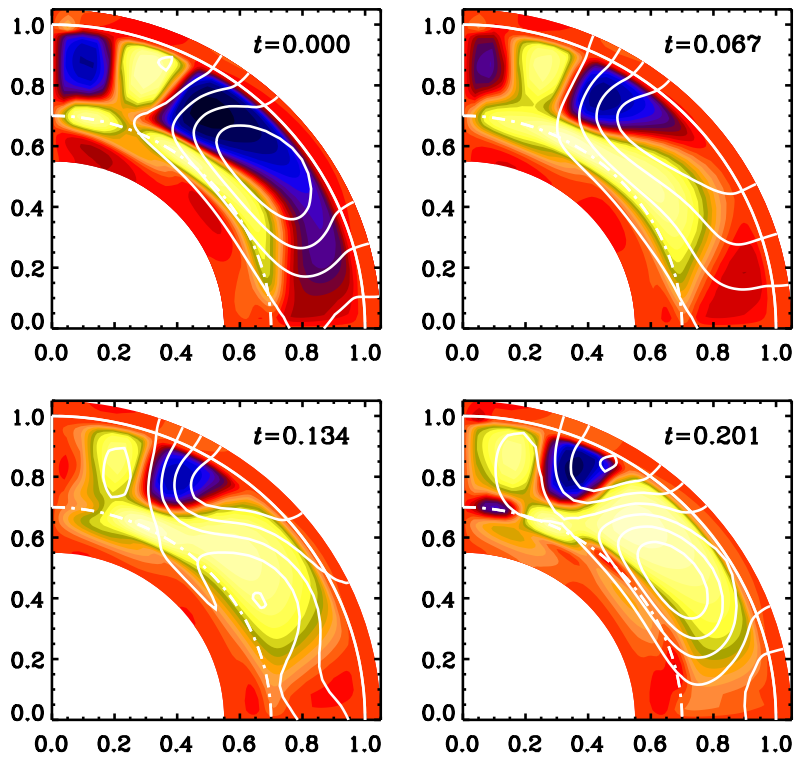

Fig. 5 Like Figure 3 but for Run K0N with $c_{\Omega 2}=0$. Note that the time $t=0$ corresponds here to a similar moment at $t=0.075$ in Figure 3. Thus, the last time at $t=0.201$ in the present figure is close to the negative field at $t=0$ in Figure 3

netic fields for the same two cases as in Figures 3 and 4. Note also that the magnetic field distribution is almost unchanged, regardless of the absence or presence of the NSSL. On the contrary, in the case without the $\partial \overline{\mathcal{E}} / \partial t$ equation, the rms magnetic field strength actually increases by about $25 \%$ when $c_{\Omega 2}=0$, while there is no difference when the $\partial \overline{\mathcal{E}} / \partial t$ equation is included. (The models presented in Figures [5] and 6] are, however, linear, so no rms value is given here.)

Generally, the local models have smaller-scale structures at the bottom of the layer, including a detailed radial dependence in the lower overshoot layer. Clearly, any mean-field structure on scales comparable to or below the scale of the turbulent eddies must be suspect. As expected, such structures are indeed absent in the more realistic nonlocal treatment, where the magnetic field is now much smoother in the deeper layers and there is no rapid field variation in the overshoot layer.

An interesting aspect of the models is that without the $\partial \overline{\mathcal{E}} / \partial t$ equation, the time variation is no longer sinusoidal, but there appear different frequencies that cannot be realistic, because the high frequencies disappear when nonlocality is included.

\subsection{Nonlinear runs}

The nonlocal approach presented here is readily applied to the nonlinear case; see the discussion in Section 2.3. Such models are shown in Figures 7 and 8 for the cases with and without nonlocality. Again, the
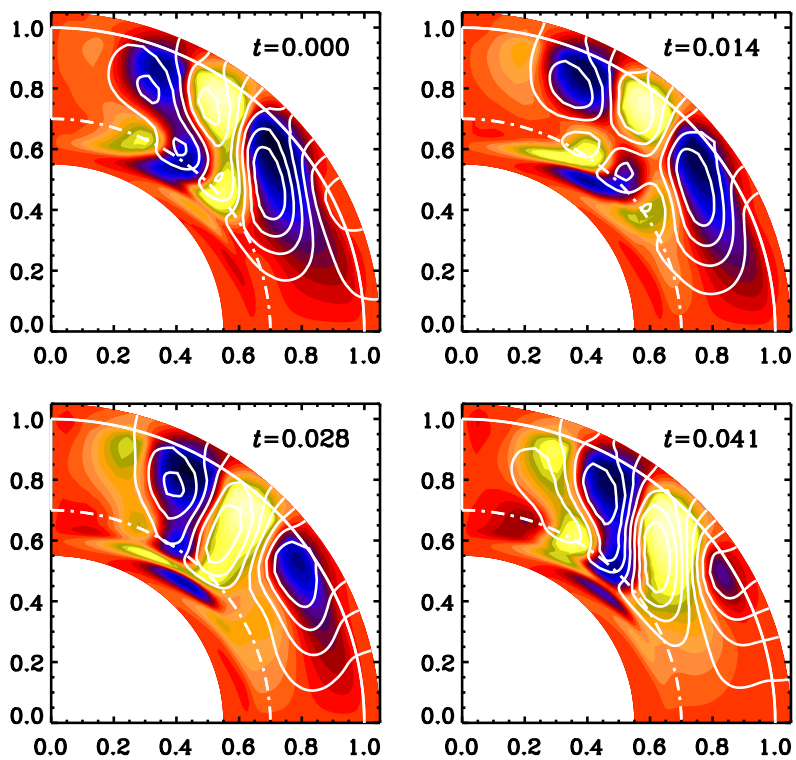

Fig. 6 Like Figure 4 but for Run KOL with $c_{\Omega 2}=$ 0 . Most of the small-scale structures appear near the bottom of the CZ, so the effect of the NSSL in Figure 4 is not so strong.

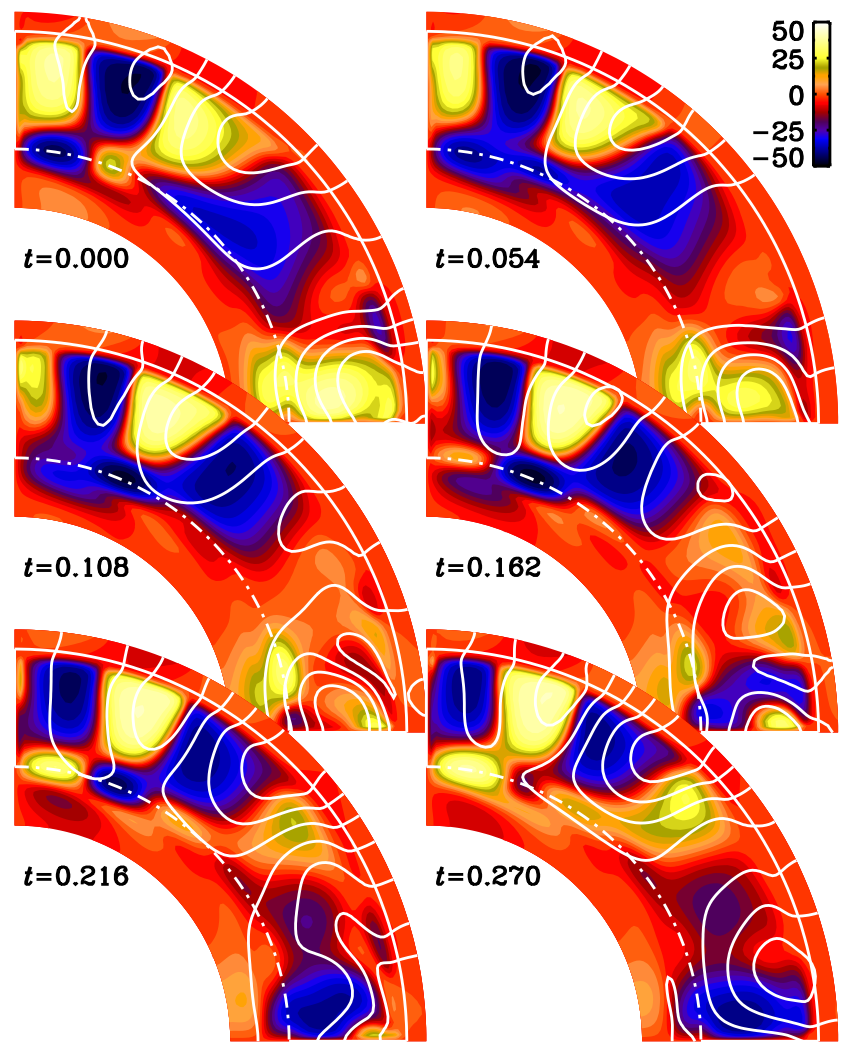

Fig. 7 Meridional cross-sections of the magnetic field of Run Q1N with $D=100$, St $=1$, and $a_{\mathcal{E}}=1$.

main difference between the two models is in the presence of small-scale structures at the bottom of the $\mathrm{CZ}$. 


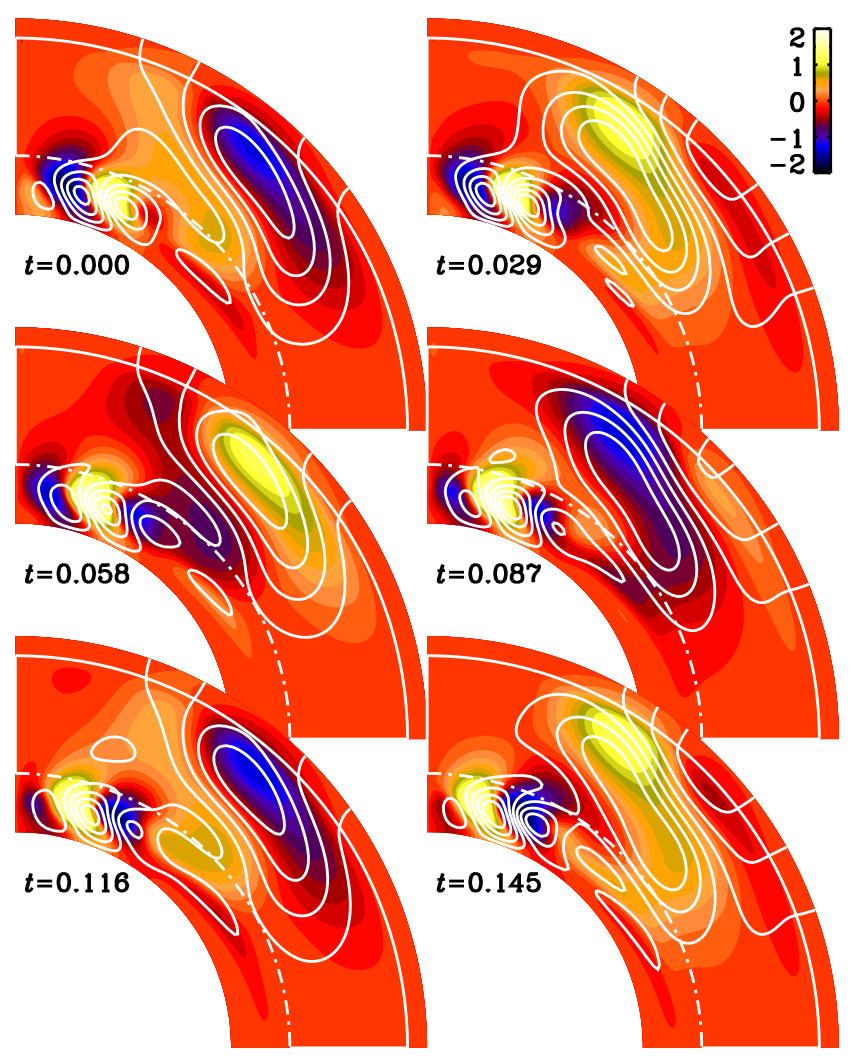

Fig. 8 Meridional cross-sections of the magnetic field of Run Q1L with $D=2500, \mathrm{St}=0$, and $a_{\mathcal{E}}=0$.
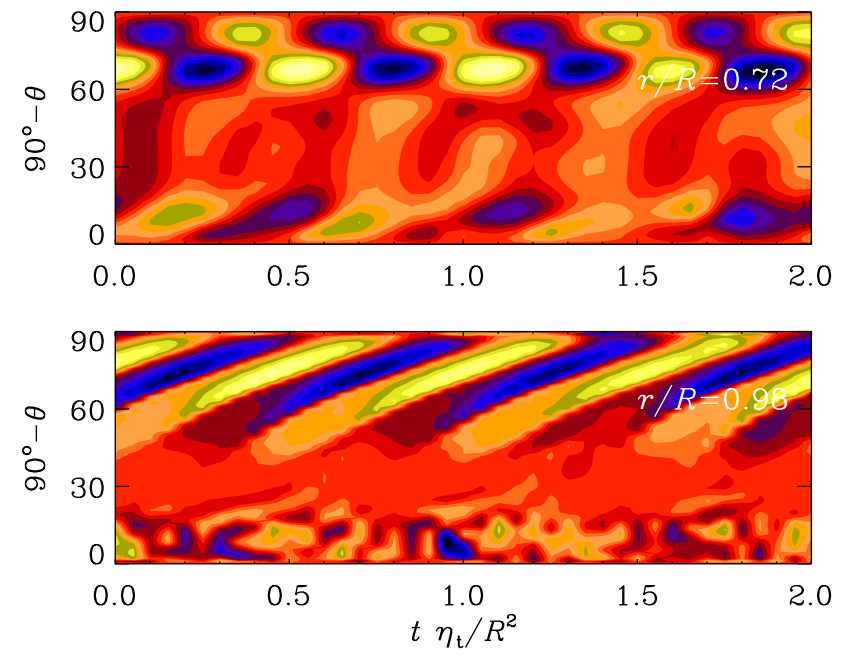

Fig. 9 Butterfly diagrams at $r / R=0.72$ (top) and 0.98 (bottom) for Run Q1N, i.e., the same run as in Figure 7 with dynamical quenching.

As a result, the overall magnetic field appears much smoother in Figure 7 than in Figure 8 As in the kinematic case, the field is strongest at high latitudes, but the nonlinear model now allows smaller-scale structures at low latitudes (Figure 7) that are not observed in the kinematic case (Figure 3).
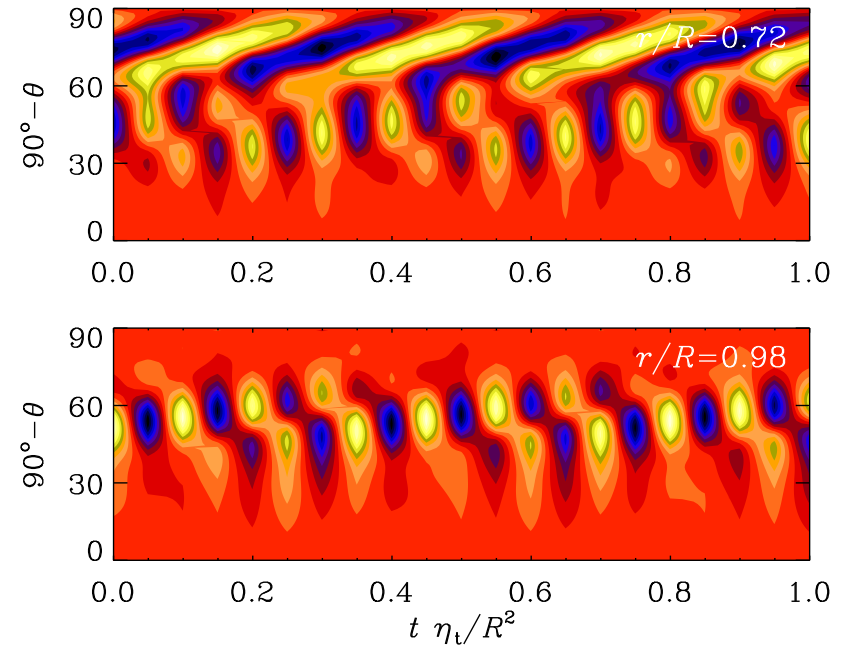

Fig. 10 Like Figure 4 , but with dynamical quenching. Butterfly diagram for Run Q1L.

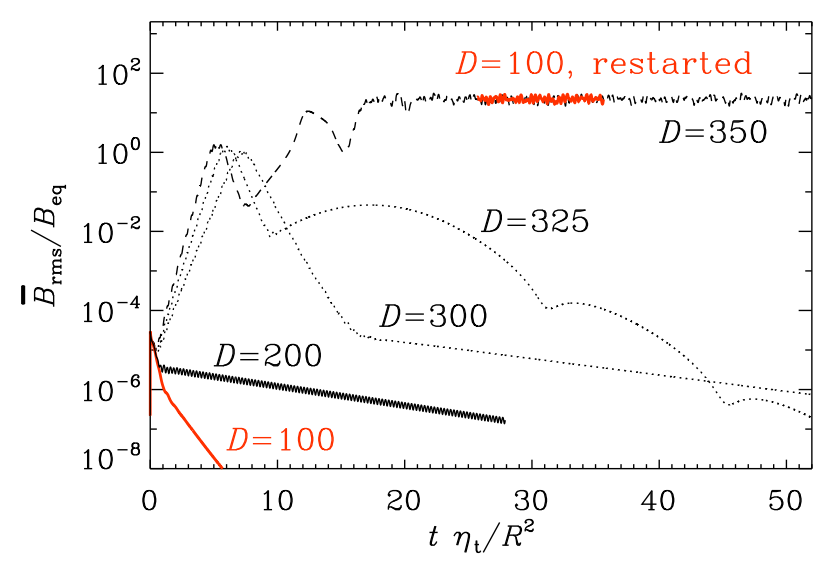

Fig. 11 Time series of runs for different values of $D$. The run with $D=350$ is supercritical, while all other runs $(D \leq 325)$ are subcritical. Two realizations of Run Q1N with $D=100$ are shown in red; the upper one has been restarted from the run with $D=350$ at $t \eta_{\mathrm{t}} / R^{2} \approx 25$ and has a nearly unchanged rms magnetic field.

Finally, we compare for the two cases in Figures 9 and 10, respectively, butterfly diagrams at the bottom of the CZ (upper panels, $r / R=0.72$ ) and the top (bottom panels, $r / R=0.98$ ). It is now clear that artificial small-scale structures are now produced in the local model, especially at the surface (Figure 10).

\subsection{Catastrophic quenching}

The dynamical quenching runs discussed in Section 3.3 have all been restarted from an earlier run. However, it turns out that their bifurcations from the trivial solution $(\boldsymbol{B}=\mathbf{0})$ are subcritical, i.e., they are finite amplitude solutions and cannot be obtained from just an infinitesimal weak seed magnetic field. To demonstrate 
this, we show in Figure 11 the time series of runs for different values of $D$.

For $D=325$ and below, all runs decay due to catastrophic quenching, because their magnetic helicity flux is too weak, and so the resulting $\alpha_{\mathrm{M}}$ profile never reaches the distribution found for a saturated dynamo. Only for a strong initial magnetic field is one able to arrive at a regime of strong supercritical magnetic helicity fluxes. This idea was first proposed by Vishniac \& Cho (2001) and later confirmed with onedimensional mean-field simulations by Brandenburg \& Subramanian (2005b), who used a prescription for the magnetic helicity fluxes from Vishniac \& Cho (2001). However, as shown in Hubbard \& Brandenburg (2011), this contribution to the magnetic helicity fluxes cannot be confirmed by direct numerical simulations, where a turbulent-diffusive magnetic helicity fluxes was found to operate instead.

\section{Conclusions}

This paper has demonstrated the great ease with which nonlocality in space and time can be implemented in a mean-field model in spherical coordinates. Unlike earlier work, we have here assumed that the nonlocality parameters $\tau$ and $\ell$ are functions of position. With this it is clear that we have entered a more speculative regime. Indeed, simulations have so far only been carried out in the case where the turbulence is statistically steady and homogeneous, so that $\tau$ and $\ell$ could be obtained as constant parameters over the whole domain (Rheinhardt \& Brandenburg 2012).

The present results appear quite plausible in many respects. In particular, while with conventional models small-scale structures appear at the bottom of the CZ, they now have been washed out when nonlocality is enabled. This is reasonable because such structures were always regarded as artifacts. One such example where this caveat was discussed was given by Chatterjee et al. (2011); see their Figures 10 and 11. Moreover, in addition to allowing for nonlocality, our approach also allows for nonlinearity in a straightforward fashion, as was already pointed out by Rheinhardt \& Brandenburg (2012).

Interestingly, Hotta et al. (2016) showed that the magnetic energies in their dynamo simulations first decreased and then increased with increasing the Reynolds number from 30 to 3000 , i.e., the dynamo was first got quenched and then recovered as more and more smaller scales were resolved in the simulation. Recently, simulation results of Hotta et al. (2016) were combined with some more simulations along the lines of the dynamo model of Fan \& Fang (2014) to calculate the values of $\alpha_{\mathrm{K}}$ and $\alpha_{\mathrm{M}}$ and interpret the behavior of the magnetic energy as being due to small-scale magnetic helicity driving (Mei Zhang, private communication).
Obviously, our approach does no longer derive from first principles, unlike standard mean-field dynamo theory which is a rigorous theory within the regime of applicability (e.g., for small magnetic Reynolds numbers). Nevertheless, we feel that in practice, when the turbulent eddies are no longer small compared with the scale over which the mean field varies, our approach is more realistic than the conventional one where nonlocality is neglected altogether. It is not obvious whether nonlocality is able to produce decisively different solutions compared with the usual dynamo solutions. It would be important to explore a wider range of models, aiming for realistic setups applied not only to the Sun, but also to galactic and accretions disc dynamos for which a number of three-dimensional simulations are already available.

One of the surprising results of global convectivelydriven dynamo simulations is the occurrence of equatorward migration in the nonlinearly saturated regime in direct numerical simulations that has not yet been explained (Käpylä et al. 2012; Augustson et al. 2015; Strugarek et al. 2017). A possible explanation for this behavior might be the occurrence of a secondary dynamo wave driven by current helicity in the nonlinear regime. To model this phenomenon correctly, nonlocality in space in necessary to prevent the formation of artificial small-scale structures. However, subsequent work of Warnecke et al. (2014) demonstrated that the cause of the equatorward migration is actually a peculiar feature in the differential rotation profile at intermediate latitudes, which they referred to as "tongues" and that the migration direction follows simply from the Parker-Yoshimura rule (Parker 1955, Yoshimura 1975). On the other hand, Augustson et al. (2015) proposed that the equatorward migration is not related to peculiar features in the differential rotation, but that it is actually a consequence of nonlinearity.

It would be useful to allow for more realistic flows that include meridional circulations and differential rotation generated in a more self-consistent manner using the $\Lambda$ effect (Rüdiger 1980, 1989), i.e., a mean-field parameterization of the Reynolds stress in the averaged momentum equation; see Brandenburg et al. (1992) and Rempel (2005) for models in that direction. Again, nonlocality should not be neglected here either. Indeed, it appears now natural to apply a similar procedure and replace the usual parameterization of the Reynolds stress by an evolution equation with a diffusion term, similar to Equation (4). The same applies also to stellar mixing length theory where such a treatment of nonlocality would seem to be able to deal in a natural way with convective overshoot, for example.

Acknowledgements. This research was supported in part by the NSF Astronomy and Astrophysics Grants Program (grant 1615100), and the University of Colorado through its support of the George Ellery Hale visiting faculty ap- 
pointment. We acknowledge the allocation of computing resources provided by the Swedish National Allocations Committee at the Center for Parallel Computers at the Royal Institute of Technology in Stockholm. This work utilized the Janus supercomputer, which is supported by the National Science Foundation (award number CNS-0821794), the University of Colorado Boulder, the University of Colorado Denver, and the National Center for Atmospheric Research. The Janus supercomputer is operated by the University of Colorado Boulder. The input files as well as some of the output files of the simulation are available under http://www .nordita.org/ brandenb/projects/spherical-

\section{References}

Augustson, K., Brun, A. S., Miesch, M., \& Toomre, J. 2015, ApJ, 809, 149

Blackman, E. G., \& Field, G. B. 2002, Phys. Rev. Lett., 89, 265007

Blackman, E. G., \& Field, G. B. 2003, Phys. Fluids, 15, L73

Brandenburg A. 2016, ApJ, 832, 6

Brandenburg, A., Käpylä, P., \& Mohammed, A. 2004, Phys. Fluids, 16, 1020

Brandenburg, A., Moss, D., \& Tuominen, I. 1992, A\&A, 265,328

Brandenburg, A., Rädler, K.-H., \& Schrinner, M. 2008a, $A \mathscr{E} A, 482,739$

Brandenburg, A., \& Subramanian, K. 2005a, Phys. Rep., 417,1

Brandenburg, A., \& Subramanian, K. 2005b, Astron. Nachr., 326, 400

Brandenburg, A., Svedin, A., \& Vasil, G. M. 2009, MNRAS, 395,1599

Brandenburg, A., \& Tuominen, I. 1988, Adv. Space Sci., 8, 185 189Variation of magnetic fields and flows during the solar cycle

Canuto, V. M., \& Mazzitelli, I. 1991, ApJ, 370, 295

Chatterjee, P., Guerrero, G., \& Brandenburg, A. 2011, $A \& A, 525$, A5

Chatterjee, P., Nandy, D., \& Choudhuri, A. R. 2004, A\&AA, 427, 1019

Choudhuri, A. R., Schüssler, M., \& Dikpati, M. 1995, A\& A, 303, L29

Del Sordo, F., Guerrero, G., \& Brandenburg, A. 2013, MN$R A S, 429,1686$

Dikpati, M., \& Charbonneau, P. 1999, ApJ, 518, 508

Fan, Y. \& Fang, F. 2014, ApJ, 789, 35

Hotta, H., Rempel, M., \& Yokoyama, T. 2016, Science, 351, 1427

Hubbard, A., \& Brandenburg, A. 2009, ApJ, 706, 712

Hubbard, A., \& Brandenburg, A. 2011, ApJ, 727, 11

Hubbard, A., \& Brandenburg, A. 2012, ApJ, 748, 51

Käpylä, P. J., Mantere, M. J., \& Brandenburg, A. 2012, ApJL, 755, L22

Krause, F., \& Rädler, K.-H. 1980, Mean-field Magnetohydrodynamics and Dynamo Theory (Oxford: Pergamon Press)

Krivodubskii, V. N. 1984, Sov. Astron., 28, 205

Madarassy, E. J. M., \& Brandenburg, A. 2010, Phys. Rev. E, 82, 016304

Mitra, D., Käpylä, P. J., Tavakol, R., \& Brandenburg, A. 2009, A\&A, 495, 1
Parker, E. N. 1955, ApJ, 122, 293

Parker, E. N. 1979, Cosmical magnetic fields (Clarendon Press, Oxford)

Pipin, V. V. 2008, Geophys. Astrophys. Fluid Dyn., 102, 21

Pipin, V. V. 2017, MNRAS, 466, 3007

Pipin, V. V., \& Kosovichev, A. G. 2011, ApJ, 727, L45

Rädler, K.-H. 1976, in Basic Mechanisms of Solar Activity, Proceedings from IAU Symposium No. 71 held in Prague, Czechoslovakia, ed. V. Bumba and J. Kleczek (D. Reidel Publishing Company Dordrecht), 323

Rädler, K.-H. 1986, Astron. Nachr., 307, 89

gêmpel, M. 2005, ApJ, 622, 1320

nileinhardt, M., \& Brandenburg, A. 2012, Astron. Nachr., 333, 71

Rüdiger, G. 1980, Geophys. Astrophys. Fluid Dyn., 16, 239

Rüdiger, G. 1989, Differential rotation and stellar convection: Sun and solar-type stars (Gordon \& Breach, New York)

Rüdiger, G., \& Hollerbach, R. 2004, The magnetic universe (New York: Wiley-VCH, Weinheim)

Stix, M. 2002, The Sun: An introduction (Springer-Verlag, Berlin)

Strugarek, A., Beaudoin, P., Charbonneau, P., Brun, A. S., \& do Nascimento, J.-D. 2017, Science, 357, 185

Subramanian, K., \& Brandenburg, A. 2006, ApJ, 648, L71

Sur, S., Brandenburg, A., \& Subramanian, K. 2008, MN$R A S, 385, \mathrm{~L} 15$

Vishniac, E. T., \& Cho, J. 2001, ApJ, 550, 752

Vitense, E. 1953, Z. Astrophys., 32, 135

Warnecke, J., Käpylä, P. J., Käpylä, M. J., \& Brandenburg, A. $2014, A p J L, 796, \mathrm{~L} 12$

Yoshimura, H. 1975, ApJS, 29, 467 